\title{
Review: evidence is lacking about suicide prevention in young people
}

\author{
Gould MS, Greenberg T, Velting DM, Shaffer D. Youth suicide risk and preventive interventions: A review of the past 10
} years. I Am Acad Child Adolesc Psychiatry 2003;42:385-405.

\section{QUESTION: What is the evidence on suicide prevention in young people?}

\section{Design}

Systematic review with narrative synthesis.

\section{Data Sources}

Studies were identified by searching PsychINFO, Medline, ERIC and Education Full Text; reference lists were hand searched.

\section{Study selection}

Population-based cross sectional studies, longitudinal studies and controlled prevention or intervention studies were included.

\section{Data extraction}

Data on suicide rates, risk factors and school based, community based and health care based prevention programs were described. Quantitative data extraction was not systematically performed, owing to the qualitative nature of many of the primary studies.

\section{Main results}

Risk factors for suicide include psychopathology, a family history of psychopathology or suicide, adverse life events, abuse and poor socio-economic circumstances. Protective factors include family cohesion and religious belief.

Schools based prevention programmes: limited evidence supports training of school staff to recognise students at risk of suicide, and crisis intervention after a suicide to reduce the risk of subsequent suicides in peers. There is insufficient evidence on the effectiveness of schoolbased suicide awareness programmes, peer support programmes, or problem-solving and coping skills development courses. Screening strategies for suicide risk yield many false positives, and youths who screen negative may become high-risk in future, suggesting that frequent repeat screening would be needed.

Community-based prevention programmes: evidence is lacking about the effectiveness of crisis helplines for youth at risk of suicide, or for the effectiveness of legislation to restrict firearms. There is evidence that implementing media guidelines to reduce "sensational" reporting of suicides reduces risk of imitation.

Health-care-based prevention and treatment interventions: educational training programmes for health professionals increase the chances of the professional asking young people about suicidal ideation, but repeated interventions are required to maintain this activity and encourage appropriate management. Crisis interventions for suicidal adolescents are based on consensus rather than randomised studies, and include reducing access to methods of suicide in the home (firearms and medicines) and "no suicide" contracts. Dialectical behaviour therapy reduces suicide among depressed adolescents and reduces subsequent suicide attempts in adults with a history of attempted suicide. Effectiveness of antidepressants has been extrapolated from adult studies, because there has been little research in adolescents. Antidepressants are now more commonly used in adolescents and may account for the recent fall in suicide rates.

\section{Conclusions}

Research is lacking about interventions to prevent suicide in young people. It seems likely that multifaceted approaches to suicide prevention and treatment are likely to be more effective than single interventions. These approaches include education, training, reducing access to methods of suicide, recognition of people at high risk and adequate treatment of depression.
Sources of funding:

Not stated.

Correspondence to: Dr Gould, Division of Child and Adolescent Psychiatry, NYSPI, 1051 Riverside Drive, Unit 72, New York NY 10032. email:

gouldm@

childpsych.columbia.edu

\section{COMMENTARY}

Morbidity and mortality for suicidal behaviour; ideation, deliberate self-harm, non-lethal attempts and completed suicide, is a substantial medical issue in the western world. The rates of youth suicide waxed then waned during the past decades and it remains difficult to know the causes of either phase.

Service utilisation and treatment costs are substantial. Clinical risk assessment and community-screening procedures are not optimal and interventions although common, sometimes have little support in the research literature. Moreover, interventions may be deployed in clinical (primary, secondary and tertiary), school, family or community populations and directed either at high-risk individuals or risk factors in the community.

Most of our knowledge about risk factors for suicidal behaviour comes from observational studies of community, school and clinical populations. The necessary ethical considerations for suicide-related research mean that experimental designs like randomised clinical trials will require considerable expertise and expense to perform. Hard endpoints, like completed suicide are low prevalence events and so experimental power can be problematic. Intermediate endpoints like suicidal ideation are more easily studied but are probably poor proxies for suicide attempt or completed suicide. Studies that combine ideation, attempt and completions into one outcome "suicidal behaviour", are thereby difficult to interpret.

Clinical implications for practice? Defining high-risk subjects in clinical populations is an important task and this has been informed by knowledge of risk and protective factors. The problem of low specificity and "false-positives" is well known. However, it is also difficult to translate the contribution of multiple risk factors for a given individual into decisions for treatment. One of the more intrusive yet common interventions, psychiatric hospitalisation, needs research evaluation. Interventions directed at risk factors have had some success with several interventions shown to be effective, at least for reducing the exposure to the risk factor. ${ }^{1}$ Implementation of these should follow.

Dr Gregory Carter, MB BS FRANZCP Cert Child Psych Head, Suicide Prevention Research Unit and Conjoint Senior Lecturer Centre for Mental Health Studies, Faculty of Health, University of Newcastle, Newcastle, Australia.

1 Burns JM, Patton GC. Preventive interventions for youth suicide: a risk factor-based approach. Aust New Zealand J Psychiatry 2000; 34: 388-407. 